\title{
Uma ontologia para a Gestão do Conhecimento no Processo de Desenvolvimento de Produto
}

\author{
Ontology for the Knowledge Management \\ in the Product Devevolpment Process
}

\author{
Maria Teresinha Tamanini Andrade ${ }^{1}$ \\ Cristiano Vasconcelos Ferreira ${ }^{2}$ \\ Hernane Borges de Barros Pereira ${ }^{3}$
}

\begin{abstract}
Resumo: As organizações necessitam gerenciar o conhecimento utilizado em seus processos de forma efetiva para promover o aprendizado organizacional e preservar seu capital intelectual. A Gestão do Conhecimento tem um papel fundamental no desenvolvimento de produtos como agente disseminador de informações para os atores envolvidos neste processo. O Processo de Desenvolvimento de Produto (PDP) tem natureza interdisciplinar e é caracterizado pelo elevado número de informações que são geradas e manipuladas. Portanto, neste processo, o compartilhamento de conhecimento, assim como a integração entre os recursos humanos, fazem-se necessários para a solução de problemas. Ontologias, enquanto sistemas de representação do conhecimento, são importantes para apoiar a organização, classificação, representação, recuperação e difusão do conhecimento no PDP. O objetivo deste artigo é descrever a elaboração de uma proposta de Gestão do Conhecimento utilizando ontologias, que apoie a representação, recuperação e disseminação de conhecimento relativo ao PDP. Para tanto, este artigo descreve um método para a formalização e construção de ontologias para subprocessos do PDP. Na sequência, apresenta o método para a construção da ontologia e sua aplicação no SENAI CIMATEC - Centro Integrado de Manufatura e Tecnologia. Palavras-chave: Gestão do conhecimento. Ontologia. Processo de Desenvolvimento do produto. Melhoria de processos.
\end{abstract}

\begin{abstract}
Organizations need to effectively manage the knowledge used in their processes for promoting organizational learning and preserving their intellectual capital. Knowledge Management plays a key role in product development as a dissemination agent of information to the actors involved in this process. Product Development Process (PDP) has an interdisciplinary nature and is characterized by the high amount of information generated and manipulated. In PDP, knowledge sharing and the integration between human resources are necessary for problem solving. Ontology as a knowledge representation system is important to support the organization, classification, representation, retrieval, and dissemination of knowledge in the PDP. This paper describes the preparation of a proposal for Knowledge Management using ontologies, which support the representation, retrieval, and dissemination of knowledge in the PDP. Hence, the proposed method to formalize and build ontologies of PDP sub-processes is described. In addition, an application carried out at SENAI CIMATEC - Center for Integrated Manufacturing and Technology is presented.
\end{abstract}

Keywords: Knowledge Management. Ontology. Product Development Process. Process Improvement.

\section{Introdução}

As organizações necessitam gerenciar o conhecimento utilizado em seus processos de forma eficiente e efetiva para promover o aprendizado organizacional e preservar seu capital intelectual. Diversos são os tipos e fontes de conhecimento, dentro e fora da organização.
Sendo assim, propostas de Gestão de Conhecimento devem ser definidas e implementadas para transformar o conhecimento individual ou de grupo em conhecimento organizacional, adquirir conhecimento de fontes externas quando necessário, apoiar a representação,

\footnotetext{
${ }^{1}$ Instituto Federal de Educação, Ciência e Tecnologia da Bahia (IFBahia), Rua Emídio dos Santos, s/n - Barbalho, Salvador - BA, Brasil, E-mail: tamanini@ifba.edu.br

${ }^{2}$ Universidade Federal de Santa Catarina - Campus Joinville, Campus Universitário, Bom Retiro, Joinville - SC, Brasil, E-mail: cristianovferreira@joinville.ufsc.br

${ }^{3}$ Programa de Modelagem Computacional, SENAI Cimatec, Av. Orlando Gomes 1845, CEP 41650-010, Salvador - BA, Brasil, Departamento de Ciências Exatas, Universidade Estadual de Feira de Santana, Campus Universitário, CEP 44031-460, Feira de Santana - BA, Brasil, E-mail: hbbpereira@gmail.com
}

Recebido em 11/8/2009 — Aceito em 28/5/2010

Suporte financeiro: Nenhum. 
recuperação e disseminação do conhecimento e, desta forma, garantir que o conhecimento utilizado e criado por membros da organização, durante a execução dos seus processos, está sendo gerenciado adequadamente (ANDRADE, 2005).

A Gestão do Conhecimento tem um papel fundamental no desenvolvimento de produtos como agente disseminador de informações para os atores envolvidos neste processo.

Clark e Fujimoto (1991) definem desenvolvimento de produto como o processo pelo qual uma organização transforma dados sobre oportunidades de mercado e possibilidades técnicas em bens e informações para a fabricação de um produto comercial.

Segundo Ferreira (2003), o Processo de Desenvolvimento de Produto (PDP) tem natureza interdisciplinar e uma de suas características é o elevado número de informações que são geradas e manipuladas. Os atores envolvidos no processo enfrentam problemas que podem ser amenizados ou resolvidos com uma eficiente Gestão do Conhecimento, tais como: dificuldade da busca e recuperação de um documento específico, devido à grande quantidade de documentos, de diferentes tipos, que são gerados ou utilizados ao longo do PDP; dificuldade de sistematizar as informações geradas ao longo do PDP, para, posteriormente, transformá-las em conhecimento agregado pela equipe de projeto; dificuldade para reutilizar o conhecimento gerado em um projeto em outro, por falta de vocabulário comum; dificuldade para identificar os profissionais com as competências desejadas para execução das atividades; perda de capital intelectual da organização, devido à rotatividade das equipes e a não representação/explicitação do conhecimento.

Portanto, neste processo, o compartilhamento de conhecimento, assim como a integração entre os recursos humanos, fazem-se necessários para a solução de problemas. A Gestão do Conhecimento é altamente propícia ao PDP devido à alta rotatividade, aliada à necessidade de agilidade nos processos $\mathrm{e}$ de inovação

Nesse contexto, sugere-se que ontologias, enquanto sistemas de representação do conhecimento, são importantes para apoiar a organização, classificação, representação, recuperação e difusão do conhecimento no PDP. Ontologia é uma especificação formal e explícita de uma conceitualização compartilhada (GRUBER, 1993). Sob uma perspectiva pragmática e técnica, a ontologia provê uma dimensão adicional de classificação que permite a recuperação de conhecimento independentemente dos processos organizacionais. Além disso, a ontologia estabelece um vocabulário comum imprescindível ao compartilhamento de conhecimento.

$\mathrm{O}$ objetivo deste artigo é descrever um método para a formalização e construção de uma ontologia para subprocessos do PDP que auxilie a Gestão do Conhecimento de processos organizacionais. Comenta-se brevemente uma experiência em Gestão de Conhecimento em uma das áreas do Centro Integrado de Manufatura e Tecnologia do Serviço Nacional de Aprendizagem Industrial (SENAI CIMATEC), de modo a observar, avaliar e validar o método proposto. O SENAI CIMATEC é uma organização cujas atividades podem ser resumidas na atividade de repassar conhecimento tecnológico para a indústria, oferecendo produtos, serviços técnicos e tecnológicos e pesquisa aplicada.

A assunção filosófica interpretativa favoreceu o desenvolvimento da pesquisa, uma vez que permitiu que o fenômeno social restrito ao contexto organizacional (i.e. a gestão do conhecimento usando ontologia como meio de tornar mais eficaz o PDP) fosse estudado com base não apenas em documentos técnicos, como também em observações in loco e aplicação de questionários.

Iniciou-se a pesquisa com a seguinte questão: É possível usar um sistema de representação do conhecimento, especificamente ontologias, para gerenciar de maneira mais eficaz os processos de classificação e recuperação do conhecimento sobre PDP de modo a melhorar todo o processo produtivo? A seguinte assertiva foi apresentada como hipótese: O uso de ontologia como sistema de representação do conhecimento aumenta a eficácia na/da gestão do conhecimento organizacional sob a perspectiva do PDP, assim como do processo produtivo.

Para realização deste trabalho, foi proposto um processo para implantação de Gerência do Conhecimento em uma organização. Este processo foi utilizado para realizar uma análise diagnóstica da Gerência do Conhecimento no SENAI CIMATEC em uma de suas áreas. Esta análise diagnóstica indicou que o objetivo específico da Gerência do Conhecimento na organização é documentar o conhecimento explícito já existente, em particular, o conhecimento sobre o PDP. Desta forma, parte deste processo foi modelada, capturando conhecimento e oferecendo uma dimensão para classificação dos documentos criados e/ou utilizados ao longo do processo. Além disso, uma ontologia foi elaborada para fornecer uma dimensão adicional para a classificação dos documentos: a interoperabilidade semântica. Como resultado do processo de implantação de Gerência do Conhecimento no SENAI CIMATEC, foi definido um conjunto de componentes para o Sistema de Gerência do Conhecimento da organização, assim como elaborado e avaliado um protótipo do componente contendo o conceito, o modelo de um processo ou a descrição de uma atividade e suas subatividades.

Este trabalho é organizado da seguinte forma: em seguida a esta introdução, são descritos os conceitos de Gestão do Conhecimento, ontologias e PDP. 
Na sequência, é apresentado um método para a formalização e construção da ontologia para um subprocesso do PDP, o Projeto Detalhado do Produto, sua aplicação em uma das áreas do SENAI CIMATEC e finalmente as conclusões do artigo.

\section{Gestão do conhecimento}

O termo Gestão do Conhecimento implica a utilização de mecanismos que auxiliem as organizações a gerenciar o conhecimento como um ativo que promova o desenvolvimento organizacional. A Gestão do Conhecimento procura aproximar o homem das tecnologias da informação, potencializando a capacidade cognitiva humana dentro de um âmbito organizacional.

O conhecimento é definido por Davenport e Prusak (1998) como sendo uma mistura fluida de experiência condensada, valores e informação contextual, que proporciona uma estrutura para a avaliação e incorporação de novas experiências e informações. Ele tem origem e é aplicado na mente dos colaboradores. O conhecimento organizacional é o conhecimento tácito e explícito de cada organização. Nas organizações, ele costuma estar embutido não só em documentos e repositórios, mas também em rotinas, processos, práticas e normas organizacionais.

Para esclarecer o que é conhecimento, Davenport e Prusak (1998) diferenciam quatro elementos: dado, informação, conhecimento e ação. Cada um destes elementos funciona como base para a existência do elemento seguinte. Os dados representam fatos distintos e objetivos, relativos a eventos ocorridos em uma organização. Já as informações são dados dotados de relevância e propósito, exercendo alguma influência sobre o julgamento do indivíduo que as utiliza. Por sua vez, conhecimento tem embutido em si valores como sabedoria e insights. Por fim, a ação se concretiza quando um funcionário utiliza em uma situação prática o conhecimento adquirido.

De acordo com Nonaka e Takeuchi (1997), o conhecimento pode ser classificado em conhecimento tácito e conhecimento explícito. O conhecimento tácito é físico, subjetivo, proveniente da experiência, específico ao contexto e difícil de ser formalizado e comunicado. $\mathrm{O}$ conhecimento explícito pode ser estruturado e verbalizado, sendo facilmente transportado, armazenado e compartilhado em documentos e sistemas computacionais.

Nonaka e Takeuchi (1997) destacam que uma organização não pode criar conhecimento sem indivíduos. Portanto, a organização deve apoiar os indivíduos criativos e lhes proporcionar um ambiente favorável para a criação do conhecimento. A criação do conhecimento organizacional deve ser entendida como um processo que amplia para a esfera da empresa o conhecimento criado pelos indivíduos, tornando-o parte da rede de conhecimentos da organização.
$\mathrm{Na}$ criação do conhecimento organizacional, os autores definem os quatro modos de conversão do conhecimento:

- Socialização - conversão do conhecimento tácito em conhecimento tácito, isto é, consiste no compartilhamento de experiências pela observação, imitação e prática, segundo o modelo mestre-aprendiz;

- Exteriorização - conversão do conhecimento tácito em conhecimento explícito por meio do uso de metáforas, analogias, conceitos, hipóteses ou modelos;

- Combinação - conversão do conhecimento explícito em conhecimento explícito, isto é, envolve a reconfiguração das informações existentes por meio da classificação, do acréscimo, da combinação e da categorização do conhecimento explícito; e

- Interiorização - conversão do conhecimento explícito em conhecimento tácito. Este modo está intimamente relacionado ao "aprender fazendo" e ocorre sob a forma de modelos mentais ou know-how técnico compartilhado.

Assim, as informações devem estar organizadas para serem acessadas quando requisitadas, necessitando estarem estruturadas e classificadas. Segundo Villela (2004), taxonomias e ontologias desempenham papel fundamental para apoiar a estruturação e classificação das informações. Taxonomias definem classes e subclasses de objetos e são mais simples que ontologias, sendo mais fáceis de serem definidas, porém, capturam menos semântica. Por outro lado, vários autores defendem o uso de ontologias na classificação do conhecimento organizacional, pois, além de auxiliarem na classificação e indexação, facilitam a busca semântica e a distribuição de conhecimento relevante, a comunicação entre múltiplos usuários e a associação entre múltiplas bases de conhecimento. Assim, a ontologia adotada deve permitir que os indivíduos da organização tenham acesso ao conhecimento que precisam ou desejam.

Alguns trabalhos de pesquisa têm sido desenvolvidos com o objetivo de auxiliar a Gestão do Conhecimento no PDP, entre eles Gouvinhas et al. (2004), Brasil e Forcellini (2004), Alliprandini e Toledo (2003) e Ferreira e Forcellini (2003).

Para melhor compreensão de como a Gestão do Conhecimento atua na melhoria do PDP, desde a criação do conhecimento até a sua utilização, Gouvinhas et al. (2004) desenvolveram um modelo que é uma forma simplificada de visualizar os processos necessários para a criação do conhecimento organizacional, sua manutenção, disseminação e utilização. 
Brasil e Forcellini (2004) apresentam uma proposta de estrutura conceitual que estabelece diretrizes para a concepção de um modelo de Gestão do Conhecimento aplicável ao PDP.

Alliprandini e Toledo (2003) apresentam uma proposta de modelo para gerência do desenvolvimento de produto a partir da discussão de aspectos críticos, atividades e tarefas, técnicas e ferramentas, organização e liderança, indicadores de desempenho, tomada de decisão, integração, comunicação e colaboração, habilidades técnica, organizacional e comercial, aprendizagem organizacional e Gestão do Conhecimento.

Ferreira e Forcellini (2003) apontam, ainda, alguns aspectos importantes para a Gestão do Conhecimento no PDP, tais como: modelagem do conhecimento, conhecimento sobre o ciclo de vida do projeto do produto, apoio às primeiras fases do PDP e reutilização do conhecimento. Estes aspectos têm grande potencial como temas para trabalhos futuros na área de Gestão do Conhecimento aplicada ao PDP.

\section{Ontologia}

Apresenta-se, a seguir, uma definição sucinta de ontologia, assumida por muitos autores, estabelecida por Thomas R. Gruber de modo a respaldar a discussão proposta.

Uma ontologia é uma especificação explícita de uma conceituação. O termo é tomado por empréstimo da filosofia, onde uma ontologia é um relato sistemático da Existência. Para sistemas baseados em conhecimento, o que 'existe' é exatamente aquilo que pode ser representado. Quando o conhecimento de um domínio é representado em um formalismo declarativo, o conjunto de objetos que podem ser representados é chamado o universo do discurso. Este conjunto de objetos, e as relações formalizadas entre eles, são refletidas no vocabulário representativo com o qual um programa baseado em conhecimento representa conhecimento. Portanto, nós podemos descrever a ontologia de um programa através da definição de um conjunto de termos representativos. Em tal ontologia, definições associam os nomes de entidades no universo do discurso (e.g. classes, relações, funções ou outros objetos) com texto legível para humanos, descrevendo o que os nomes significam e axiomas formais que restringem a interpretação e o uso bem formado destes termos. (GRUBER, 1993, p. 199)

Como observado, as ontologias fornecem um vocabulário compartilhado e facilitam o entendimento do universo que modelam, favorecendo, consequentemente, a comunicação entre os diversos usuários e a associação entre várias bases de conhecimento.
Para O’Leary (2001), uma ontologia é uma descrição de conceitos e relações que podem existir para um agente ou uma comunidade de agentes. $\mathrm{O}$ termo ontologia foi adotado da Filosofia. Há muito tempo, filósofos têm usado ontologias para tentar descrever domínios naturais e a existência dos seres e das coisas em si. Esse termo foi recentemente adotado também pelas comunidades de inteligência artificial e gestão do conhecimento referindo-se a conceitos e termos que podem ser usados para descrever alguma área do conhecimento ou construir uma representação desse conhecimento.

Alguns métodos para a construção de ontologias foram propostos, como em Uschold e King (1995) e Grüninger e Fox (1995). É desejável reunir, em uma única proposta, as melhores características dos métodos apresentados. Com esta finalidade, Falbo (1998) propôs um método para a construção de ontologias. Esse método é basicamente usado nesta pesquisa para construir uma ontologia que servirá como suporte para a Gestão do Conhecimento no PDP. As principais fases deste método são descritas a seguir.

- Propósito e Especificação de Requisitos: tem como propósito identificar a competência da ontologia, que diz respeito à cobertura de questões que a ontologia deve responder. Assim, ao se estabelecer as questões de competência de uma ontologia, tem-se um meio eficaz de identificar o que é relevante ou não;

- Captura da Ontologia: tem por objetivo identificar e organizar os conceitos e relações relevantes. Conceitos devem ser definidos utilizando linguagem natural e exemplos. Deve-se, ainda, construir taxonomias, de modo a organizar hierarquicamente as categorias e subcategorias. Axiomas devem ser providos para definir a semântica dos termos. Os axiomas especificam definições de termos na ontologia e restrições sobre sua interpretação;

- Formalização da Ontologia: a linguagem natural muitas vezes introduz incertezas e ambiguidades. Por isso, a necessidade de utilização de uma linguagem formal, que, por meio de seus símbolos não ambíguos e formulações exatas, conduza a uma maior clareza e correção nas deduções;

- Integração de Ontologias Existentes: visa aproveitar conceituações anteriormente estabelecidas;

- Avaliação da Ontologia: a ontologia deve ser avaliada com base nas questões formais de competência, na sua especificação de requisitos e/ou no mundo real; e

- Documentação da Ontologia: a documentação deve incluir propósitos, requisitos, descrições 
textuais da conceituação, ontologia formal e critérios de projeto.

Para apoiar a construção de ontologias, Leite e Breitman (2004) propuseram um processo baseado no Léxico Ampliado da Linguagem (LAL). O LAL é uma linguagem de representação simples, composta de três entidades: termo, noção e impacto, cujo objetivo é mapear o vocabulário utilizado no domínio.

O processo de construção baseado no LAL é estruturado e segue princípios de engenharia de software e técnicas já estabelecidas para a captura, modelagem e posterior validação da informação modelada. O processo leva em conta as tarefas de elicitação, modelagem e análise para explicitar e comunicar o conhecimento do domínio. Este processo mapeia os termos do LAL nos elementos da ontologia. Os termos do tipo objeto e sujeito são mapeados em conceitos; os termos do tipo verbo são mapeados em propriedades; os termos do tipo estado são mapeados em conceitos ou propriedades; a noção de cada termo é mapeada na descrição do respectivo conceito ou propriedade; por fim, por meio da lista de impactos de cada termo do léxico, mapeia-se o verbo em propriedades e o predicado em restrições dos conceitos. A ontologia resultante será expressa por meio das suas primitivas básicas, isto é, conceitos, propriedades, hierarquia (de conceitos), restrições e axiomas.

Para guiar e avaliar a construção de ontologias é necessário ter critérios de qualidade objetivos e fundamentados no propósito do produto resultante. Uschold e King (1995) enumeraram um conjunto de critérios para avaliar a qualidade de uma ontologia. Estes critérios, relacionados a seguir, devem nortear o processo de construção de uma ontologia em todas as suas etapas.

- Clareza: uma ontologia deve comunicar efetivamente o significado projetado dos termos definidos e, assim, suas definições devem ser objetivas;

- Consistência: uma ontologia deve garantir consistência na sua definição, tanto dos axiomas lógicos quanto dos conceitos informais;

- Extensibilidade: a partir de uma ontologia, deve ser possível definir novos termos para usos específicos, sem haver necessidade de rever definições existentes;

- Compromissos de codificação mínimos: não deve haver dependência em relação a uma tecnologia particular de representação do conhecimento; e

- Compromissos ontológicos mínimos: uma ontologia deve fazer o mínimo de imposições possíveis, permitindo que as partes comprometidas com a ontologia fiquem livres para especializar e instanciar a ontologia.
Outro critério de qualidade bastante utilizado consiste em verificar a competência da ontologia. As questões de competência são definidas na fase de especificação da ontologia e são utilizadas para avaliar se a ontologia responde às questões para as quais foi projetada.

\section{Processo de desenvolvimento do produto}

O processo de desenvolvimento de produto pode ser estruturado sob a abordagem de processos ou sistêmica. $\mathrm{Na}$ abordagem por processos, o desenvolvimento de produtos pode ser entendido como um conjunto de atividades inter-relacionadas, como informações de entrada, atividades, ferramentas e informações de saída. Na abordagem sistêmica, o desenvolvimento de produtos assume uma dimensão maior. Os processos associados ao desenvolvimento de produto (por exemplo, definição da estratégia da empresa) são identificados, compreendidos, geridos e suas interfaces promovem um resultado eficaz na obtenção dos objetivos. Os responsáveis devem conhecer os processos da organização, controlar e melhorar estes processos de forma integrada, para obter resultados para o negócio (ROZENFELD et al., 2006).

De acordo com a Norma ISO 9001:2000, um processo é um conjunto de atividades inter-relacionadas e interatuantes que transformam entradas em saídas. Por sua vez, segundo o PMI (Project Management Institute), um projeto é um esforço temporário empreendido para criar um produto, serviço ou resultado exclusivo.

Assim, o processo de projeto de um produto pode ser entendido como sendo um conjunto de atividades e tarefas estruturadas e organizadas de forma a atingir um determinado resultado. No caso, o resultado final esperado é o produto. $\mathrm{E}$, com base em Back et al. (2008), cada atividade ou tarefa contém os seguintes elementos: i) entradas, informações ou objetos físicos a serem processados ou transformados pela tarefa; ii) saídas, informações ou objetos físicos processados ou transformados pela tarefa (entregas produzidas). Além disto, possuem mecanismos (recursos físicos e/ ou informações necessárias para a execução da tarefa (por exemplo: metodologias, técnicas, ferramentas) e controle (informações usadas para monitorar ou controlar a tarefa), entretanto serão tratados em outra dimensão.

O processo de desenvolvimento de produto apresenta algumas características intrínsecas, as quais podem ser consideradas ao longo do projeto. Estas características, mesmo que não ocorram concomitantemente, impactam no ciclo de desenvolvimento de produto e, devem ser levadas em conta ao se tratar da gestão do conhecimento. Estas características, listadas abaixo, são resultado das 
análises dos trabalhos de Pahl e Beitz (2003), Pahl et al. (2005), Rozenfeld et al. (2006), Back et al. (2008), assim como, observações das práticas industriais. São elas:

- Desenvolvimento colaborativo - partes de um produto são desenvolvidas em distintas regiões do planeta. As equipes precisam se comunicar e vencer barreiras como: idiomas, horários, recursos, culturais;

- Uso intenso de ferramentas computacionais para auxiliar o desenvolvimento do produto - estas ferramentas CAD (Computer Aided Design), CAE (Computer Aided Engineering) permitem avaliar a dinâmica, rigidez, aerodinâmica, vibração, entre outras características do produto;

- Investimentos elevados para desenvolver um novo produto - em virtude disto, as empresas adotam diferentes estratégias com parceiros de desenvolvimento/fornecedores, de forma a minimizar os riscos da atividade;

- Tempo de desenvolvimento do produto é elevado - principalmente se se considerar que, desde a definição do modelo do produto até o lançamento no mercado, podem ocorrer mudanças governamentais, econômicas e sociais que impactem nos resultados planejados com o lançamento do produto no mercado;

- Complexidade dos componentes e da interface com os clientes - este fato requer o uso intenso da tecnologia para redução de custo, redução de peso e aumento da qualidade do produto;

- Cadeia produtiva complexa e com distintas características - as empresas adotam diferentes estratégias de relacionamento com os seus fornecedores. Estes fornecedores, por sua vez, dependem de outros fornecedores. Em muitos casos, um único fornecedor é responsável por disponibilizar a mesma peça para distintos clientes;

- Clientes com diferentes perfis de consumo um mesmo produto é adquirido por clientes de diferentes regiões, com hábitos, culturas e interesses distintos. Em se tratando de produtos globalizados, os diferentes perfis de consumo tornam mais crítico o processo de desenvolvimento de produto; e

- Diferentes sistemas de vendas - impactam na forma na qual a empresa relaciona-se com o cliente e na diversidade de serviços prestados;

- Equipe forte para desenvolver produtos locais e suportar as adequações de produtos externos - com conhecimento em distintas áreas do conhecimento, experiência, capacidade de entrega, comprometida e pró-ativa.

O processo de desenvolvimento de produtos é descrito por inúmeros autores - Asimov (1962), Back (1983), VDI 2222 (1977), Pahl e Beitz (2003), Pahl et al. (2005), Rozenfeld et al. (2006), Back et al. (2008), entre outros - por meio de modelos que representam as atividades, os métodos, as técnicas e as ferramentas para o projeto em engenharia.

De forma geral, estes modelos apresentam semelhanças em suas fases e etapas. Desta forma, é possível agrupar o processo de desenvolvimento de produtos nas seguintes fases e etapas:

- Fase de Pré-Desenvolvimento do Produto, a qual busca alinhar o planejamento estratégico da empresa e o planejamento estratégico do produto e, consequentemente, o planejamento do projeto;

- Desenvolvimento do Produto, o qual envolve a definição do problema de projeto, ciclo de vida, usuários e necessidades do projeto, projeto informacional (especificação de problemas de projeto), projeto conceitual (síntese de soluções alternativas para o produto), projeto preliminar (configuração do leiaute do produto, dimensionamento, modelagem, simulação de sistemas técnicos ou de tecnologias), projeto detalhado (normatização, representação e documentação de sistemas técnicos); e

- Pós-desenvolvimento do produto, que contempla o acompanhamento do processo e do produto no mercado, retirada e descarte do produto.

O modelo descrito em Rozenfeld et al. (2006) ilustrado na Figura 1, mostra uma visão geral do processo de desenvolvimento de produto. $\mathrm{Na}$ fase de pré-desenvolvimento ocorre o planejamento estratégico do produto e planejamento do projeto. $\mathrm{Na}$ fase de desenvolvimento são realizadas as etapas de projeto informacional, conceitual, detalhado, preparação da produção e lançamento do produto no mercado. E, no pós-desenvolvimento ocorre o acompanhamento do produto e processo e descontinuidade do produto no mercado.

Sob uma perspectiva mais específica, o Processo de Desenvolvimento de Produto (PDP) do SENAI CIMATEC é apresentado na Figura 2 e constitui-se de um conjunto de atividades, procedimentos e regras que devem ser realizadas e aplicadas sistematicamente, desde a definição do problema de projeto até a solução detalhada do produto. As informações são a matéria-prima principal com a qual o projetista trabalha e, também, são os resultados a que ele chega. Por intermédio das informações, os processos são executados e os meios de projeto são aplicados, isto é, os meios são o ferramental teórico e prático 
que, disponibilizados para os projetistas, permitem que o PDP possa ser conduzido e operacionalizado, viabilizando e efetivando a obtenção de soluções de projeto. E, por meio das estruturas e das formas de representação das informações, as soluções de projeto são formalizadas. As informações estão associadas ao conhecimento de especialistas de distintos campos de conhecimentos envolvidos no projeto e nas estimativas de custos. A metodologia de projeto de produtos possui elementos metodológicos estruturados e organizados de forma a apoiar o raciocínio da equipe de projeto quando ela necessita entender e resolver um dado problema de projeto.

Em virtude da grande quantidade de informações a serem manipuladas e das distintas competências dos recursos humanos envolvidos no PDP, tornam-se necessárias a sistematização e a Gestão do Conhecimento deste processo.

O PDP pode ser beneficiado enormemente pela Gestão do Conhecimento. As atividades relacionadas com este processo têm um caráter essencialmente criativo e, portanto, dependem das habilidades e conhecimentos das pessoas envolvidas. Além disso, a produtividade é obtida pelo uso intenso de técnicas e métodos em constante evolução, os quais estão amadurecidos e são um fator decisivo para o sucesso de sua aplicação.

Para Ferreira (2004), a gestão dos conhecimentos envolvidos no desenvolvimento de produtos (e.g. engenharia de produto, engenharia de projeto de moldes, engenharia de fabricação, engenharia de processo, engenharia de materiais e engenharia de software) tem se tornado importante para as empresas na medida em que permite aumentar a quantidade de informações a serem manipuladas, melhorar a qualidade dos produtos e reduzir o tempo de desenvolvimento de produto. Devido às características do PDP, é fundamental que ocorra o compartilhamento de conhecimento tácito e explícito entre os especialistas envolvidos em suas atividades.

Neste contexto, uma eficiente Gestão do Conhecimento deve permitir que as experiências e as soluções adotadas em cada projeto possam ser difundidas por meio da organização, obtendo a melhoria contínua do processo de negócio. Para tanto, é fundamental armazenar o conhecimento sobre os processos de forma integrada. Assim, na próxima seção, é apresentado um método para a construção de uma ontologia para um subprocesso do PDP, com base na proposta de Falbo (1998).

\section{Uma ontologia para o projeto detalhado do produto}

A construção da ontologia tem por objetivo promover o compartilhamento e reuso do conhecimento sobre o Projeto Detalhado do Produto (Figura 3), um subprocesso do PDP, estruturando e representando o conhecimento existente sobre o domínio, facilitando a busca e recuperação de documentos e apoiando a aquisição de conhecimento ao longo do Projeto Detalhado do Produto. Este processo foi escolhido por ser repleto em conhecimentos tácitos e explícitos. É composto por doze atividades, que, por sua vez, são compostas de subatividades. As atividades 7 e 8, no entanto, são elementares, o que significa que não possuem subatividades. $\mathrm{O}$ conhecimento explícito é representado pelos Padrões Operacionais (PO 01) e Instruções de Trabalho (entre as quais: IT 06, IT 11 e IT 34). Relatórios, propostas, planos e especificações, tais como o Relatório de Projeto Conceitual e o Modelo 3D Revisado, constituem

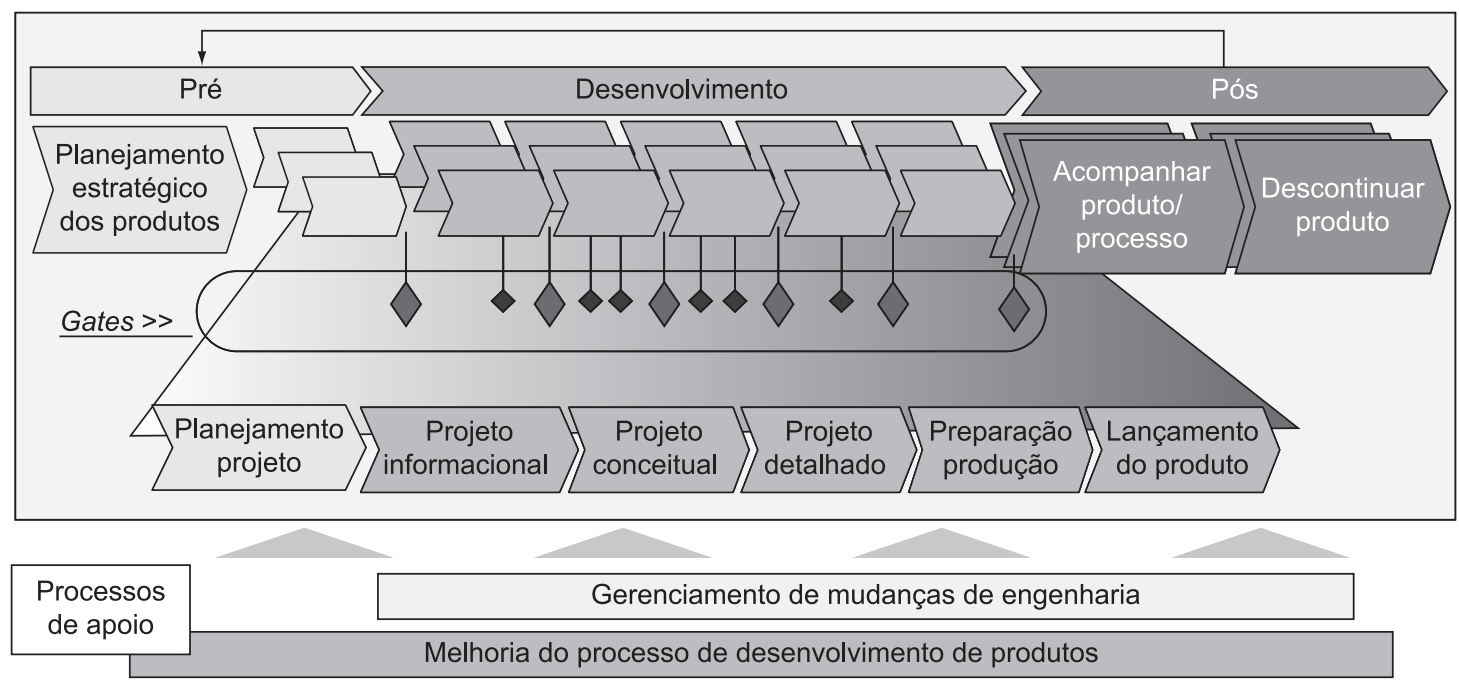

Figura 1. Modelo de desenvolvimento de produtos proposto por Rozenfeld et al. (2006). 

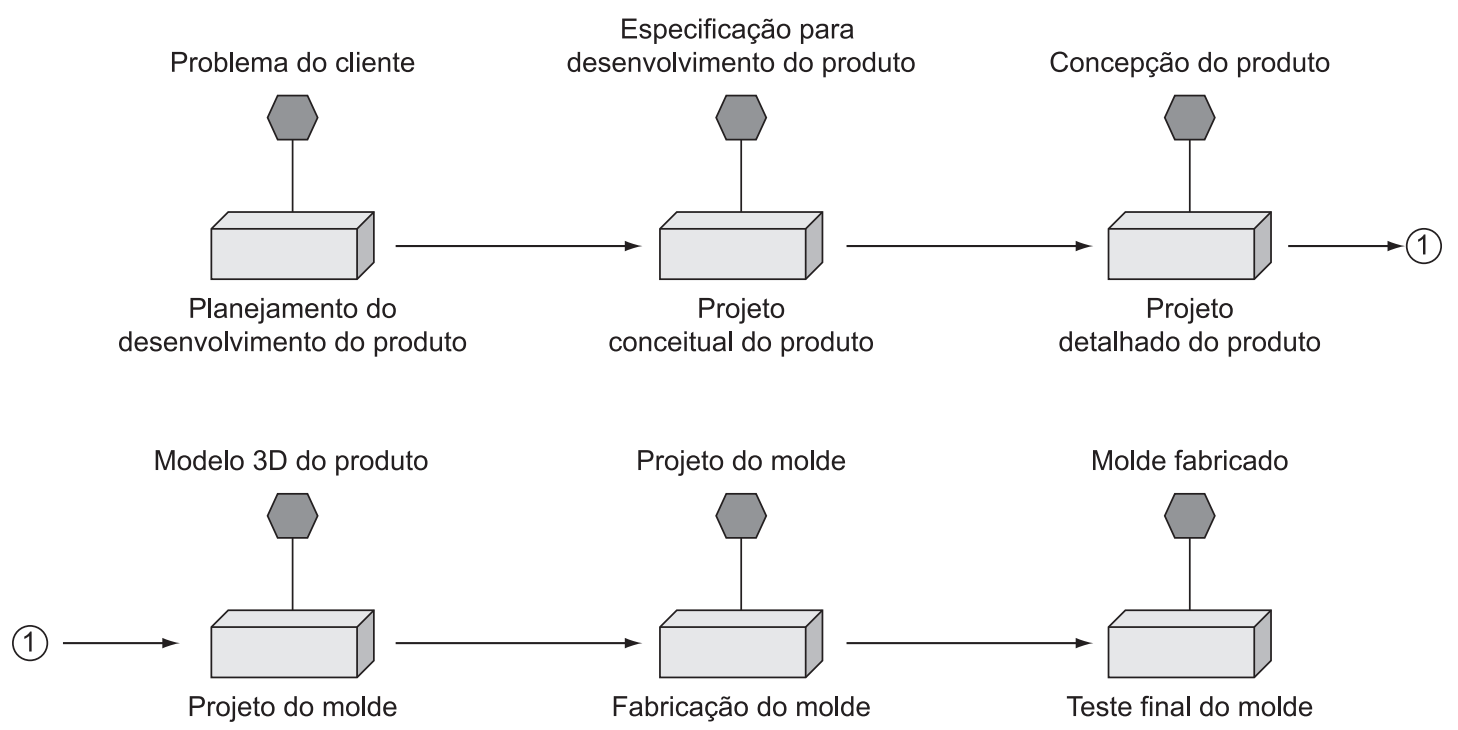

Figura 2. Processo de Desenvolvimento de Produto do SENAI CIMATEC apresentado por Andrade (2005).

os documentos utilizados e produzidos ao longo do PDP. Operações lógicas são utilizadas para indicar as possibilidades de variação das instâncias específicas de execução do processo, por exemplo: o protótipo do produto, um artefato produzido na atividade 10 , pode ser aprovado ou conter problemas originados na elaboração da solução técnica ou na análise de engenharia. Diferentes atividades serão executadas a depender da situação específica.

A seguir são apresentados os cenários de motivação, propostos por Andrade (2005), que instigaram o desenvolvimento deste trabalho e, principalmente, determinaram a utilidade da ontologia para o domínio. Segundo Uschold e King (1995), cenários de motivação são problemas ou situações que mostram a utilidade da ontologia.

- Dificuldade da busca e recuperação de um documento específico, devido à grande quantidade de documentos, de diferentes tipos, que são gerados ou utilizados ao longo do PDP;

- Dificuldade de sistematizar as informações geradas ao longo do PDP, para, posteriormente, transformá-las em conhecimento agregado pela equipe de projeto;

- Dificuldade para reutilizar o conhecimento gerado em um projeto em outro, por falta de vocabulário comum;

- Dificuldade para identificar os profissionais com as competências desejadas para execução das atividades; e

- Perda de capital intelectual da organização, devido à rotatividade das equipes e a não representação/explicitação do conhecimento.
Para a definição do escopo da ontologia, Andrade (2005), com base em Villela (2004), formulou algumas questões de competência descritas a seguir. Uschold e King (1995) definem questões de competência como questões que a ontologia deve responder.

1. Como o PDP é decomposto em processos?

2. Qual o produto ou componente de produto fornecido como resultado de um determinado processo?

3. Como um determinado processo é decomposto em atividades?

4. Quais são os insumos e recursos materiais e intelectuais necessários para executar uma determinada atividade?

5. Quais são os produtos resultantes da execução de uma determinada atividade?

6. Como são classificadas as matérias-primas utilizadas como insumo para o Projeto Detalhado do Produto?

7. Como são classificados os artefatos utilizados como insumo ou recurso, ou produzidos ao longo do Projeto Detalhado do Produto?

8. Quais são os componentes de um determinado artefato?

9. Quais são os perfis profissionais necessários para a execução de uma atividade?

10. Quais são as ferramentas de software que automatizam um determinado procedimento?

11. Quais procedimentos podem ser utilizados para executar uma determinada atividade?

12. Quais pessoas possuem uma determinada competência? 


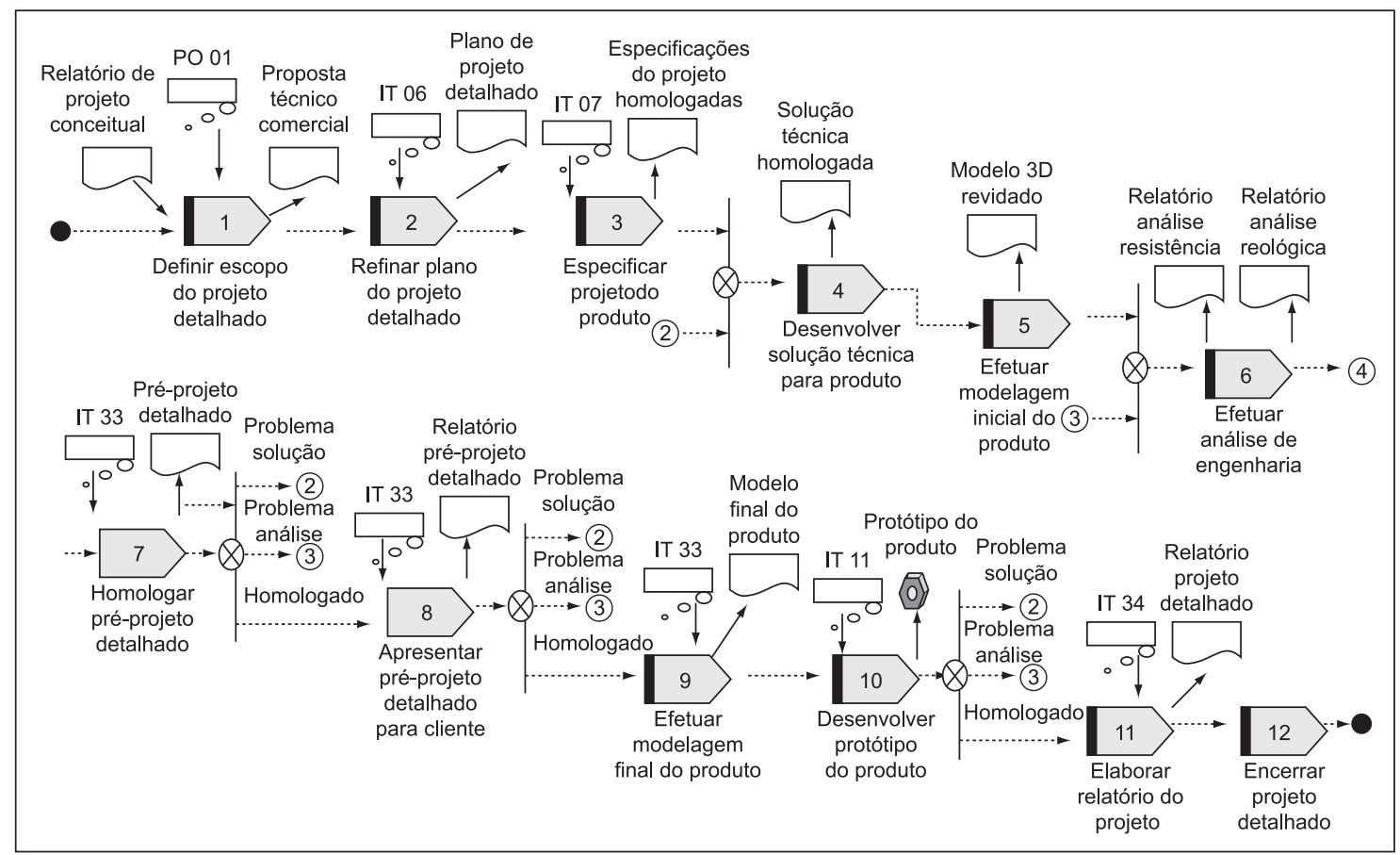

Figura 3. Processo de desenvolvimento de produto - subprocesso projeto detalhado do Produto. Fonte: Andrade (2005).

13. A que domínio de conhecimento pertence um conhecimento?

A Ontologia do Projeto Detalhado do Produto estende um subconjunto da Ontologia de Organização (VILLELA, 2004). Foi fundamentada no estudo do Manual de Desenvolvimento de Produtos do SENAI CIMATEC e em entrevistas com especialistas da área. Durante seis meses, gerentes, coordenadores e técnicos foram entrevistados em reuniões previamente agendadas, às vezes com pauta livre, outras, com pauta estabelecida para captura de conhecimentos tácitos e explícitos.

Um dos resultados e contribuições da pesquisa é a construção da ontologia para o domínio do Projeto Detalhado do Produto, dentro da qual foram definidas quatro subontologias, pois existiam conhecimentos distintos no domínio que deveriam ser agrupados conforme suas características. Assim, definiu-se a subontologia de capital intelectual, a subontologia de comportamento, a subontologia de artefatos e a subontologia de matéria-prima (Figura 4).

A subontologia de capital intelectual estabelece o vocabulário para descrever o capital intelectual que é necessário para o Projeto Detalhado do Produto tratando dos seguintes aspectos: taxonomia de competência, interação entre experiência e conhecimento, disponibilidade de competências e decomposição de domínio de conhecimento. Responde às questões de competência 12 e 13.
A subontologia de comportamento aborda os seguintes aspectos: decomposição de processos em atividades, decomposição de processo em processos, resultado de processo, decomposição de atividade, taxonomia de atividade, taxonomia de procedimento e automatização de procedimento. Responde às questões de competência de 1 a 5 e de 9 a 11 .

A subontologia de artefatos aborda os aspectos de decomposição de artefatos, taxonomia de artefato quanto ao estágio de evolução, taxonomia de artefato quanto à natureza, representação de componente ou produto funcional por desenho de engenharia e arquivamento de documento. Responde às questões de competência 2,7 e 8.

A subontologia de matéria-prima aborda o aspecto da taxonomia de matéria-prima, respondendo à questão de competência 6 .

$\mathrm{Na}$ representação da ontologia, foram elaborados modelos utilizando a Unified Modeling Language (UML). Além disso, os conceitos, relações, restrições e argumentos foram descritos em linguagem natural e exemplificados. Na formalização, foi utilizada lógica de primeira ordem, definindo-se as constantes, predicados e axiomas. A avaliação da ontologia ocorreu ao longo do seu processo de construção.

Assim, a seguir, serão apresentados alguns exemplos da formalização da ontologia para o subprocesso Projeto Detalhado do Produto. Para efeito deste artigo, será apresentada parte da subontologia de artefatos, alguns conceitos, restrições e respectivos axiomas. 
Escolheu-se esta subontologia porque foi a mais trabalhada em relação ao domínio de conhecimento estudado, o PDP.

A Figura 5 apresenta um excerto da subontologia de artefatos e como um artefato pode ser classificado quanto ao estágio de evolução.

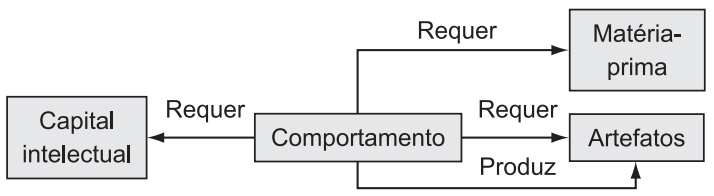

Figura 4. Mapa conceitual das subontologias da ontologia de projeto detalhado do produto.
A Figura 6 apresenta um excerto da subontologia de artefatos e como um artefato pode ser classificado quanto à sua natureza.

A taxonomia de artefato quanto à natureza envolve muitos conceitos além dos já apresentados, tendo sido necessário dividi-la em subtaxonomias para facilitar a leitura e o entendimento. Estas subtaxonomias são: subtaxonomia de bem de produção, subtaxonomia de documento e subtaxonomia de componente. $\mathrm{Na}$ Figura 7 apresenta-se um excerto da subtaxonomia de bem de produção.

Na Tabela 1 são descritos alguns dos conceitos e definidos alguns dos predicados e argumentos para a formalização da subontologia de artefatos. Argumentos são representados por variáveis, por exemplo, s, e representam os conceitos definidos na ontologia.

Tabela 1. Excertos dos conceitos da subontologia de artefatos.

\begin{tabular}{|c|c|c|}
\hline Conceito & Predicado & Descrição \\
\hline Artefatos & artefato(s) & $\begin{array}{l}\text { São quaisquer elementos produzidos pelo homem e não } \\
\text { por causas naturais, podendo exercer diferentes papéis, } \\
\text { tais como o de insumo ou produto de uma atividade. }\end{array}$ \\
\hline Artefatos finais & artefatofinal(s) & São produtos acabados e prontos para serem entregues ao clie \\
\hline Protótipos & protótipo(s) & $\begin{array}{l}\text { São versões preliminares do produto } \\
\text { representadas por um modelo físico. }\end{array}$ \\
\hline Protótipos manuais & protótipomanual(s) & $\begin{array}{c}\text { São protótipos feitos por um especialista, } \\
\text { também chamados de mockup. }\end{array}$ \\
\hline Protótipos rápidos & protótiporápido(s) & São protótipos feitos em máquina de prototipagem rápida. \\
\hline Bens de produção & bemprodução(s) & $\begin{array}{c}\text { São artefatos cujas funcionalidades apoiam a criação ou a } \\
\text { transformação de outros artefatos. }\end{array}$ \\
\hline Hardwares & hardware(s) & $\begin{array}{c}\text { São bens de produção representados por um computador, } \\
\text { um de seus periféricos ou uma máquina qualquer } \\
\text { operada com o auxílio de um software. }\end{array}$ \\
\hline
\end{tabular}

Softwares software(s)

Softwares de

planejamento e

controle

Softwares de

engenharia

\section{Softwares}

corporativos e de gestão

Equipamentos

Documentos

documento(s)

Produtos funcionais produtofuncional(s)

Componentes

swplanejcontrole(s)

swengenharia(s)

swcorporatgestão(s)

equipamento(s)
São bens de produção representados por um conjunto de instruções e dados que, utilizado em conjunto com um hardware, é capaz de executar ou apoiar a execução de atividades.

São softwares que apoiam a execução de atividades de planejamento e controle de projetos e da produção.

São softwares que apoiam a execução de atividades de engenharia. Exemplos são as ferramentas de software do tipo

CAD (Computer Aided Design), CAE (Computer Aided Engineering) e CAM (Computer Aided Manufature).

São softwares que apoiam a gestão da cooperação.

São bens de produção, sem ser um software ou hardware, requeridos para a execução de uma atividade, podendo ser uma máquina ou um instrumento qualquer.

São artefatos escritos ou que podem ser impressos, cuja função é fornecer informação, conhecimento ou prova. São artefatos resultantes de atividades de produção que têm utilidade para os clientes independente de serem utilizados em conjunto com outros produtos ou componentes. Não são bens de produção.

São artefatos resultantes de atividades de produção que representam as partes que compõem um produto funcional. 
No trabalho original, para a Ontologia de Projeto Detalhado do Produto, modelou-se um conjunto de axiomas composto por 55 (cinquenta e cinco) axiomas. Para efeito deste artigo e considerando seu escopo, foram selecionados sete axiomas referentes à subontologia de artefatos. $\mathrm{O}$ tratamento feito a estes sete axiomas se estende aos demais. A seguir, apresentam-se as restrições e os respectivos axiomas extraídos da subontologia de artefatos.

1. Todo desenho de engenharia possui uma representação;

2. Um componente só pode ser composto por outros componentes do mesmo tipo;

3. Um documento só pode ser composto por outros documentos;

4. Artefatos que são fornecidos a clientes como resultado da execução de um processo no PDP não são bens de produção;

5. Um protótipo pode ser de um produto funcional ou componente plástico;

6. Produto de componente único não tem desenho 2D de montagem, modelo 3D de montagem e mockup virtual; e

7. Rendering é gerado por técnica de rendering.

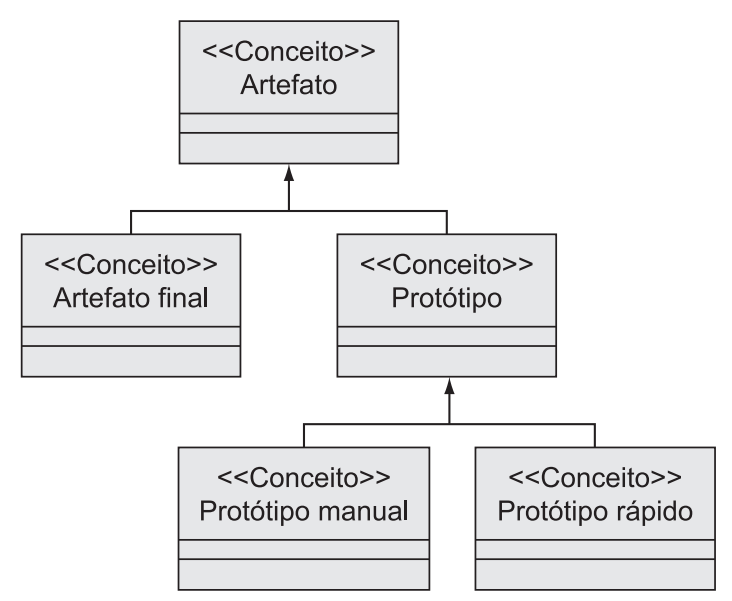

Figura 5. Subontologia de artefato: taxonomia de artefato quanto ao estágio de evolução.
Na Tabela 2 são apresentados os axiomas selecionados da subontologia de artefatos.

\section{Validação da proposta}

Para a validação da ontologia modelada a partir do método proposto, foi construído um protótipo a partir do conhecimento capturado na modelagem do subprocesso do PDP, o Projeto Detalhado do Produto.

$\mathrm{O}$ foco do protótipo foi a disponibilização do conhecimento previsto no processo, de forma a possibilitar a avaliação da abordagem adotada. $\mathrm{O}$ protótipo foi implementado como um conjunto de páginas web interligadas, em que é possível ter acesso, por meio de links, à conceitos, descrição das atividades elementares, como também aos documentos utilizados e aos roteiros dos documentos que devem ser gerados ao longo dos processos. Cada página contém o conceito, o modelo de um processo ou a descrição de uma atividade e suas subatividades.

Para avaliar a adequação da abordagem proposta com respeito à representação do conhecimento sobre os processos e a busca e recuperação de documentos associados, foi conduzida uma avaliação do protótipo por meio da aplicação de um questionário com quinze perguntas a 12 especialistas do SENAI CIMATEC, alocados em diversas atividades do Projeto Detalhado do Produto. Dos 12 especialistas, um era o coordenador da área, quatro eram técnicos de processo especializados, dois eram projetistas e cinco eram estagiários.

Treze das quinze perguntas do questionário (i.e. da 1 a 13) possuíam respostas na escala de Likert (1. Nunca; 2. Quase nunca; 3. Às vezes; 4. Quase sempre; 5. Sempre) e duas permitiam resposta aberta. As questões são listadas a seguir:

1. A forma na qual o PDP foi apresentado auxilia no entendimento do processo?

2. No modelo proposto, é possível identificar os subprocessos relacionados ao PDP?

3. No modelo proposto, é possível identificar o(s) evento(s) que provoca(m) a execução de um determinado subprocesso?

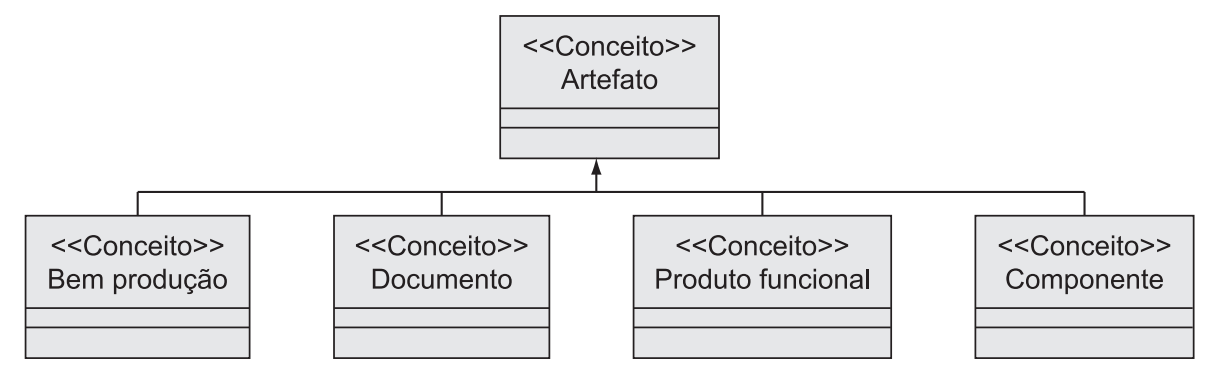

Figura 6. Subontologia de artefato: taxonomia de artefato quanto à natureza. 
4. Você entendeu como o subprocesso Projeto Detalhado do Produto é decomposto em atividades?

5. Você entendeu como são decompostas as atividades que compõem o Projeto Detalhado do Produto?

6. Foi possível compreender as atividades elementares do Projeto Detalhado do Produto?

7. Foi possível identificar os pontos de decisão existentes no Projeto Detalhado do Produto?

8. Foi possível identificar os artefatos requeridos e gerados pelas atividades ao longo do Projeto Detalhado do Produto?

9. Foi possível identificar os tipos de conhecimento (explícito e implícito ) gerados ou manipulados ao longo do Projeto Detalhado do Produto?

10. Foi possível ter acesso aos conhecimentos explícitos requeridos para execução das atividades, quando se encontram documentados?

11. Foi fácil entender o conhecimento acessado?
12. Foi possível identificar quais atores executam quais atividades do Projeto Detalhado do Produto?

13. Os nomes dados aos processos, eventos, atividades, artefatos, conhecimentos e atores auxiliam no entendimento de cada um desses elementos?

14. Quais as vantagens do modelo proposto?

15. Quais as desvantagens do modelo proposto?

A Figura 8 apresenta a síntese dos resultados da aplicação do questionário baseado na escala de Likert supracitada. Na Figura 8a, observam-se os resultados das treze questões fechadas. Não houve respostas para os itens da escala "1. Nunca" e "2. Quase nunca". Na Figura 8b, notam-se os resultados mais significativos (i.e. o somatório das respostas para os itens da escala "5. Sempre" e "4. Quase sempre") com a indicação da média (representada pelo ponto) e desvio padrão (representado pela barra de erro).

Por outro lado, após a análise das questões abertas (i.e. 14 e 15), destacamos os seguintes comentários dos especialistas do SENAI CIMATEC:

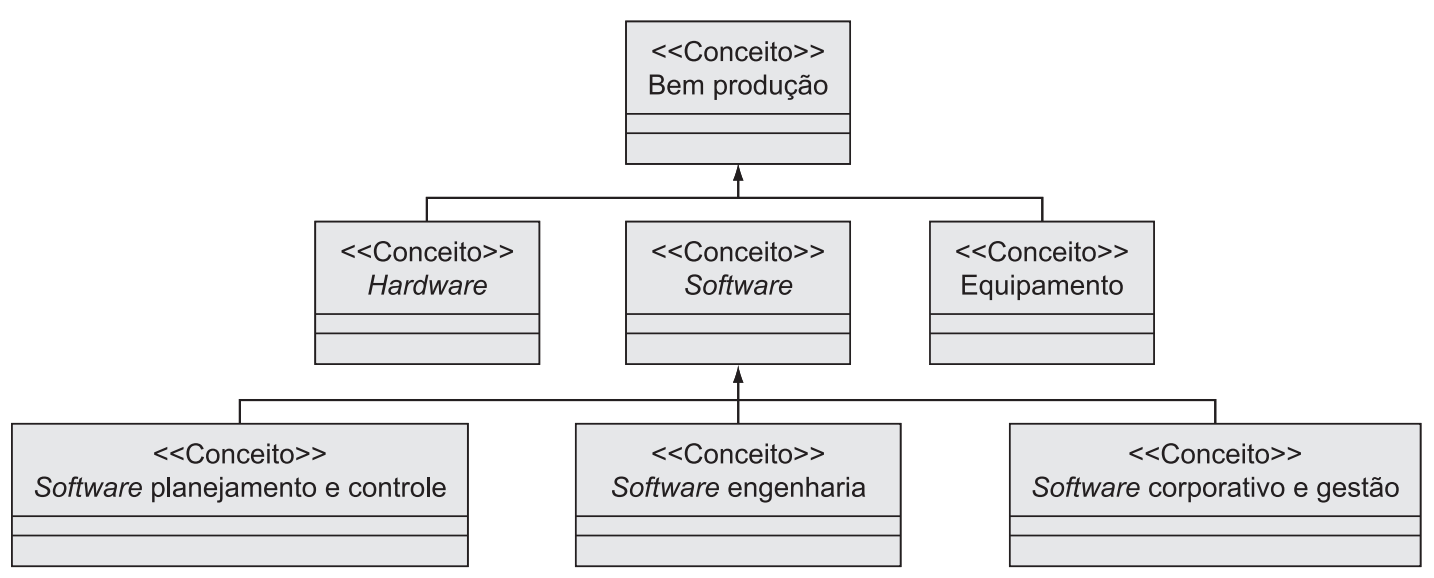

Figura 7. Taxonomia de artefato: subtaxonomia de bem de produção.

Tabela 2. Excertos dos axiomas da subontologia de artefatos.

\begin{tabular}{|c|c|c|}
\hline \# & Axiomas & Exemplo \\
\hline 1 & $(\forall d, s)$ & desenhoengenharia $(d) \rightarrow(\exists s)$ representação $(d, s)$ \\
\hline 2 & $\left(\forall s_{1}, s_{2}, t_{1}, t_{2}\right)$ & (componente $\left(s_{1}, t_{1}\right) \wedge$ componente $\left(s_{2}, t_{2}\right) \wedge$ subartefato $\left.\left(s_{1}, s_{2}\right) \rightarrow t_{1}=t_{2}\right)$ \\
\hline 3 & $\left(\forall s_{1}, s_{2}\right)$ & $\left(\right.$ documento $\left(s_{2}\right) \wedge \operatorname{subartefato}\left(s_{1}, s_{2}\right) \rightarrow$ documento $\left.\left(s_{1}\right)\right)$ \\
\hline 4 & $(\forall p, s)$ & $($ fornecimento $(s, p) \rightarrow \operatorname{processo}(p) \wedge \operatorname{artefato}(s) \wedge \neg \operatorname{bemprodução~}(s))$ \\
\hline 5 & $(\forall s)$ & $($ protótipo $(s) \rightarrow$ produtofuncional $(s) \vee$ componente $(s$, compplástico $))$ \\
\hline 6 & $(\forall d, s)$ & $\begin{array}{c}\left(\left(\text { representação }(d, s) \wedge\left(\neg \exists s_{1}\right) \text { subartefato }\left(s_{1}, s\right)\right) \rightarrow(\neg \text { desenho } 2 D(d, 2 \text { Dmontagem })\right. \\
\wedge \neg \text { modelo } 3 D(d, 3 \text { Dmontagem }) \wedge \neg \text { modelo } 3 D(\text { d,mockupvirtual })))\end{array}$ \\
\hline 7 & $(\forall s, t)$ & $\begin{array}{c}(\text { produto }(s, t) \wedge(\text { desenho } 2 D(s, 2 \text { Drendering }) \vee \operatorname{modelo} 3 D(s, 3 \text { Drendering })) \leftrightarrow \\
(\exists p)(\operatorname{adoção}(p, t) \wedge \text { tecoperacional }(p, \text { Tecrendering })))\end{array}$ \\
\hline
\end{tabular}




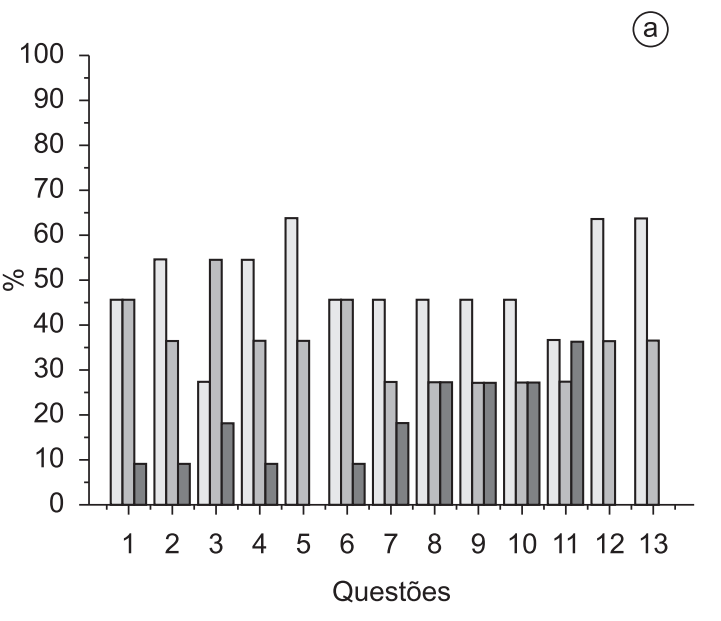

$\square$ Sempre $\square$ Quase sempre $\square$ Às vezes

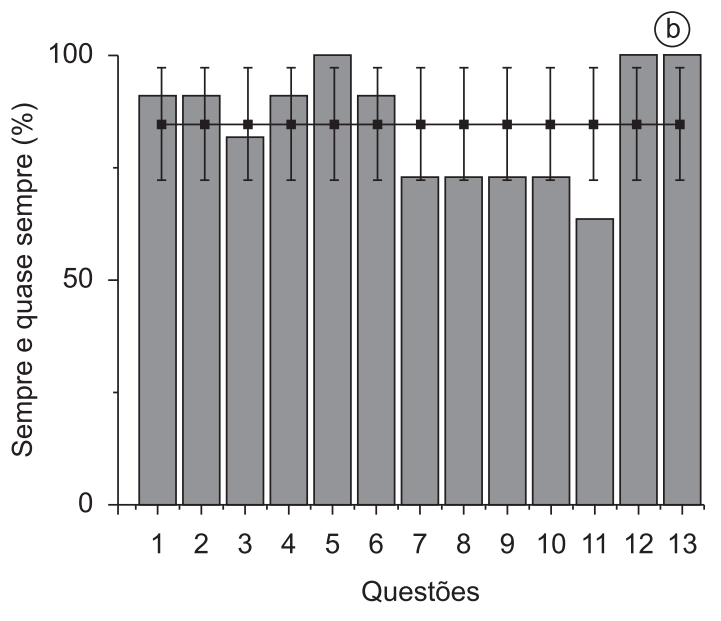

$\square$ Sempre e quase sempre $\rightarrow$ Média (desvio padrão)

Figura 8. Síntese dos resultados da aplicação do questionário baseado na escala de Likert (1. Nunca; 2. Quase nunca; 3. Às vezes; 4. Quase sempre; 5. Sempre).

- Por meio da ontologia obtêm-se os conceitos e as instâncias dos conceitos, auxiliando na compreensão do domínio estudado;

- O modelo proposto representa o PDP de forma didática, facilitando a compreensão do processo. Caso a pessoa não seja um especialista da área, pode ter uma visão mais geral e, se desejar, pode aprimorar seu entendimento por meio da exploração das subatividades;

- Há facilidade para compreender a representação sequencial das atividades e a relação com as demais atividades e subatividades;

- O acesso a documentos é importante para a realização das atividades, assim como, a identificação dos atores envolvidos em cada atividade; e

- Os conhecimentos explícitos estão claramente identificados.

Pela observação e avaliação, verificou-se que os especialistas foram capazes de identificar satisfatoriamente (Figura 8b) os subprocessos do PDP e as atividades e subatividades do Projeto Detalhado do Produto, compreender as atividades elementares do processo, identificar os atores e as atividades que eles executam, identificar os pontos de decisão, identificar os artefatos e tipos de conhecimento requeridos e gerados pelas atividades e compreender o conhecimento documentado.

Pela análise da modelagem gráfica do subprocesso Projeto Detalhado do Produto, observou-se a relação dos artefatos de saída das atividades e subatividades do subprocesso com os axiomas da Ontologia do Projeto Detalhado do Produto, mais especificamente, da subontologia de artefatos. A relação é apresentada na Tabela 3.

A ontologia e a modelagem gráfica do Projeto Detalhado do Produto auxiliaram na captura do conhecimento existente na organização a seu respeito, fornecendo uma dimensão para classificação dos conhecimentos utilizados ao longo do processo. O usuário pode buscar e recuperar documentos ao explorar os modelos de processos da organização em diferentes níveis de abstração e selecionar o documento desejado a partir do seu contexto de criação ou uso.

O objetivo do modelo de processos do PDP foi representar o conhecimento sobre o processo genérico de desenvolvimento de produto, estabelecendo as atividades e atores que devem ser considerados no planejamento de um projeto específico e, especialmente, os documentos e conhecimentos a serem gerados e/ou manipulados.

A premissa é que modelos de processos fornecem uma dimensão natural para a classificação e recuperação de conhecimento em organizações e a ontologia provê uma dimensão adicional de classificação que permite a classificação e recuperação de conhecimento. Além disso, a ontologia estabelece um vocabulário comum imprescindível ao compartilhamento de conhecimento.

Neste sentido, as conceituações específicas, que constituem o vocabulário comum estabelecido pela ontologia, por exemplo, os axiomas facilitam o desenvolvimento de futuros mecanismos para aquisição de conhecimento e preservação do capital intelectual da organização, evitando a perda de conhecimento quando membros saem da organização. Também auxiliam na promoção do aprendizado organizacional, ao facilitar o treinamento e, principalmente, facilitando 
Tabela 3. Relação dos axiomas da subontologia de artefato com os artefatos de saída das atividades e subatividades do subprocesso Projeto Detalhado do Produto.

\begin{tabular}{|c|c|c|}
\hline Axiomas & Atividades & Artefatos de saída \\
\hline A1 & $5,6,7,9,10$ & Desenho 2D, Modelo 3D \\
\hline A2 & 10 & Protótipo \\
\hline A3 & $1,2,3,4,5,6,7,8,9,11,12$ & $\begin{array}{l}\text { Comunicação, contrato, especificação, desenho de } \\
\text { engenharia, plano de projeto, proposta, relatório }\end{array}$ \\
\hline A4 & $1,2,3,4,5,6,7,8,9,10,11,12$ & $\begin{array}{l}\text { Comunicação, contrato, especificação, desenho de } \\
\text { engenharia, plano de projeto, proposta, protótipo, relatório }\end{array}$ \\
\hline A5 & 10 & Protótipo \\
\hline A6 & $5,6,7,9,10$ & Protótipo \\
\hline A7 & $5,6,7,9,10$ & Desenho 2D, Modelo 3D \\
\hline
\end{tabular}

discussões sobre os processos e conhecimentos explícitos entre os membros da organização.

A partir da proposta de Gestão do Conhecimento apresentada neste trabalho, obtiveram-se alguns benefícios, como: melhoria na execução dos processos pela diminuição da ocorrência de falhas e do apoio à tomada de decisão; preservação do capital intelectual da organização; promoção do aprendizado organizacional; aumento das vantagens competitivas da organização, pois os modelos de processos aumentam a visibilidade das mudanças a serem realizadas neles, com o objetivo de torná-los mais ágeis e/ou introduzir novas tecnologias.

\section{Considerações finais}

A crescente importância dada pelas organizações à Gestão do Conhecimento requer que sejam definidos e implementados mecanismos mais eficientes para apoiar as atividades da Gestão do Conhecimento. Neste contexto, o objetivo deste trabalho foi elaborar um modelo de Gestão do Conhecimento, construindo uma ontologia para apoiar a representação, recuperação e disseminação do conhecimento em Organizações de Desenvolvimento de Produtos.

Assim, a construção da ontologia para o Projeto Detalhado do Produto, discutida brevemente neste artigo, além de fornecer uma dimensão adicional para classificação e recuperação do conhecimento, estabelece um vocabulário imprescindível ao compartilhamento e difusão dos conhecimentos tácitos e explícitos entre os indivíduos envolvidos no PDP. A busca e recuperação de documentos podem ser feitas a partir dos conceitos e instâncias dos conceitos propostos na ontologia, utilizando os mecanismos de busca de softwares das organizações. Isto permite buscas mais eficientes por estarem baseadas no significado do termo e não simplesmente no termo.

Uma vez verificada a adequação da abordagem e do conhecimento disponibilizado, faz-se necessária a melhoria da infraestrutura técnica utilizada e a captura do conhecimento sobre os demais subprocessos do PDP. A melhoria da infraestrutura técnica é fundamental para que os modelos possam evoluir de acordo com as necessidades organizacionais, e envolve implementar componentes para descrição de processos com mecanismos para a gerência dos modelos de processo, ou seja, mecanismos para inclusão, alteração e exclusão de elementos e modelos.

Alguns trabalhos podem ser desenvolvidos com o propósito de melhorar e estender o estudo apresentado, por exemplo: modelar os demais subprocessos do PDP e os demais processos da organização; estender a ontologia de acordo com as necessidades dos demais subprocessos do PDP e dos demais processos da organização; pesquisar e/ou desenvolver os componentes para definição e instanciação de ontologias e gerência de competências; desenvolver ferramentas para apoio à manutenção do conhecimento, garantindo que o conhecimento adquirido não se torne defasado e não haja redundâncias de conhecimento no repositório da organização.

\section{Referências}

ALLIPRANDINI, D. H.; TOLEDO, J. C. Modelo para gestão do processo de desenvolvimento de produtos: uma proposta baseada em dimensões críticas. In: CONGRESSO BRASILEIRO DE GESTÃO E DESENVOLVIMENTO DE PRODUTOS, 4, 2003, Gramado, RS, Brasil. Anais...

ANDRADE, M. T. T. Uma proposta para Gestão do Conhecimento ao longo do Processo de Desenvolvimento de Produto. Dissertação (Mestrado em Redes de Computadores) - Unifacs, Salvador, 2005.

ASIMOV, M. Introduction to design: fundamentals of engineering design. [S.1.]: Prentice Hall, 1962. (Traduzido para o português: Introdução ao projeto de engenharia. São Paulo: Mestre Jou, 1968).

BACK, N. Metodologia de projeto de produtos industriais. Rio de Janeiro: Guanabara Dois, 1983.

BACK, N. et al. Projeto Integrado de Produtos: Planejamento, Concepção e Modelagem. [S.1.]: Editora Manole, 2008. 
BRASIL, A. D.; FORCELLINI, F. A. Diretrizes para a concepção de um modelo envolvendo a gestão do conhecimento no processo de desenvolvimento de produtos. Santa Catarina: Departamento de Engenharia Mecânica, Universidade Federal de Santa Catarina UFSC, 2004.

CLARK, K. B.; FUJIMOTO, T. Product development performance: strategy, organization and management in the world auto industry. Boston-Mass: Harvard Business School Press, 1991.

DAVENPORT, T. H.; PRUSAK, L. Conhecimento Empresarial: como as organizações gerenciam o seu capital intelectual. Rio de Janeiro: Campus, 1998.

FALBO, R. Integração de Conhecimento em um Ambiente de Desenvolvimento de Software. Tese (Doutorado) COPPE, Universidade Federal do Rio de Janeiro - UFRJ, Rio de Janeiro, 1998.

FERREIRA, C. V. Metodologia para as Fases de Projeto Informacional e Conceitual de Componentes de Plásticos Injetados Integrando os Processos de Projeto e Estimativa de Custos. Tese (Doutorado em Engenharia Mecânica) - Universidade Federal de Santa Catarina - UFSC, Santa Catarina, 2004.

FERREIRA, M. G.; FORCELLINI, F. Gestão do Conhecimento no Processo de Desenvolvimento de Produto: visão do presente e futuro. In: CONGRESSO BRASILEIRO DE GESTÃO E DESENVOLVIMENTO DE PRODUTOS, 4, 2003, Gramado, RS, Brasil. Anais... GOUVINHAS, R. P. et al. Aplicação da Gestão do Conhecimento no Processo de Desenvolvimento de Produtos. São Paulo: KMBRASIL, 2004.
GRUBER, T. R. A translation approach to portable ontologies. Knowledge Acquisition, v. 5, n. 2, p. 199-220, 1993.

GRUNINGER, M.; FOX, M. S. Methodology for the Design and Evaluation of Ontologies. Toronto: University of Toronto, 1995. Technical Report.

LEITE, J.; BREITMAN, K. Ontologias: Como e porquê criá-las. In: CONGRESSO DA SOCIEDADE BRASILEIRA DE COMPUTAÇÃO, 24, 2004, Salvador, BA.

NONAKA, I.; TAKEUCHI, H. Criação do conhecimento na empresa: como as empresas japonesas geram a dinâmica da inovação. Rio de Janeiro: Campus, 1997.

O'LEARY, D. How Knowledge Reuse Informs Effective System Design and Implementation. IEEE Intelligent Systems, 2001.

PAHL, G.; BEITZ, W. Engineering Design: A Systematic Approach. [S.1.]: Editora Springer Verlag, 2003.

PAHL, G. et al. Projeto na Engenharia: Fundamentos do desenvolvimento eficaz de produtos, métodos e aplicações. São Paulo: Edgard Blücher, 2005.

ROZENFELD, H. et al. Gestão de Desenvolvimento de Produtos: Uma referência para a melhoria do processo. [S.1.]: Editora Saraiva, 2006.

USCHOLD, M.; KING, M. Towards a Methodology for Building Ontologies. In: WORKSHOP ON BASIC ONTOLOGICAL ISSUES IN KNOWLEDGE SHARING, 1995.

VDI 2222. Konstruktionsmethodik, konzipieren technischer Produkte. Düsseldorf: VDI - Verlag, 1977.

VILLELA, K. Definição e construção de ambientes de desenvolvimento de software orientados a organização. Tese (Doutorado) - Universidade Federal do Rio de Janeiro - UFRJ, Rio de Janeiro, 2004. 